derived from the GMMH Unlicensed Medicines Policy, previous audits of HDAT use and the RCPsych consensus report on HDAT prescription.

Result. 11 of 252 patients (4\%) were identified as being on HDAT, of which eight were due to polypharmacy and three to high dose of a single antipsychotic. For $1 / 11$ patients target symptoms and a risk/benefit rationale were documented. The mean length of time on HDAT was 6 years. 7/11 patients had either tried or considered clozapine in the past. 8/11 patients had not had an ECG within the last year, $4 / 11$ had not had yearly U\&E. 8/11 had regular mental health reviews.

Conclusion. Compliance with the audit standards was found to be highly variable. This may reflect many factors, including the length of time since commencing HDAT and the complex shared care arrangements currently in place in Trafford. Thus, the following recommendations have been made:

To start a register of all patients prescribed HDAT.

To review local guidelines and documentation to ensure they are up to date and can be effectively implemented in routine clinical practice.

To ensure that the responsibility for conducting yearly physical health checks for patients prescribed HDAT is communicated to the relevant parties.

Patient factors associated with the use of psychotropic polypharmacy in patients under the care of a community mental health team in the West of Ireland

Karthika Srikumar ${ }^{1 *}$, Richard Walsh ${ }^{2}$, Donnchadh Walsh ${ }^{2}$, Sonn Patel ${ }^{1}$ and Sheila O'Sullivan ${ }^{1}$

${ }^{1}$ Acute Adult Mental Health Unit and ${ }^{2}$ School of Medicine, University College Dublin

${ }^{*}$ Corresponding author.

doi: 10.1192/bjo.2021.314

Aims. Psychiatric polypharmacy refers to the prescription of two or more psychotropic medications to any one patient. This definition is purely quantitative and does not take into account whether such a prescription is detrimental, or unnecessary. In many cases, polypharmacy has been implemented in challenging illnesses, and some studies have shown that it can improve overall outcomes for certain patients. Evidence suggests that the prevalence of psychotropic polypharmacy is increasing, despite advances in psychosocial interventions. The aim of this study was to assess the current prevalence of polypharmacy among patients being treated by a community mental health team (CMHT), and the patient factors associated with its use.

Method. We performed a cross-sectional study of all patients registered with a CMHT in a mixed urban/rural area on a single date. Case records were examined to determine the most recently prescribed drug regimen for each patient. Clinical chart diagnoses were recorded and each one independently verified by the team consultant using ICD-10. A number other sociodemographic variables were recorded. Using Microsoft Excel, we analysed the medications prescribed as well as rates and levels of polypharmacy based on multiple different patient characteristics.

Result. Of the 245 patients, the mean age was 56.3 and $51.2 \%$ $(\mathrm{n}=126)$ were female. Psychotropic polypharmacy was seen in $62 \%(n=152)$ of patients. $33 \%(n=82)$ of patients were on two psychotropic medications, and of this subset, a combination of one antipsychotic and one antidepressant was the most common drug regimen, seen in $16.7 \%(n=41)$ of all patients.
Polypharmacy was more prevalent in females, with $68 \%(\mathrm{n}=85)$ being on two or more psychotropics, in comparison to $58 \%$ of male patients. In relation to age, patients aged between 51 to 65 years had the highest prevalence of polypharmacy, at a rate of $71 \%(\mathrm{n}=49)$. Among all primary diagnoses, polypharmacy was most common in patients with affective disorders, with $80 \%$ (n $=40$ ) of this patient cohort on two or more medications. Second to this was psychotic disorders, with polypharmacy seen in $65 \%(n=62)$ of this group.

Conclusion. We found that psychotropic polypharmacy is highly prevalent in psychiatric patients being treated in a community setting. Certain demographics and patient factors, such as age, gender and psychiatric diagnosis influenced the rate of polypharmacy and certain drug combinations were more commonly prescribed than others.

\section{Monitoring side-effects of antipsychotics using the glasgow antipsychotic side-effect scale}

James Sterritt*

Dorset Healthcare University NHS Foundation Trust

${ }^{\star}$ Corresponding author.

doi: 10.1192/bjo.2021.315

Aims. Antipsychotic drugs frequently produce side-effects which represent common reasons for noncompliance. National guidelines, published by the National Institute of Care and Health Excellence, the Royal College of Psychiatrists, and the Maudsley Prescribing Guidelines in Psychiatry, stipulate that patients prescribed antipsychotic drugs should be reviewed for side-effects on a weekly basis. This completed audit cycle, conducted on a mixed acute general adult psychiatric ward, examined whether patients were being assessed for side-effects of antipsychotic drugs using a standardised, self-reporting scale - the Glasgow Antipsychotic Side-effect Scale (GASS) - as per national guidelines. As identification of side-effects is important in tailoring treatment to improve compliance, auditing monitoring practice was important in realising these outcomes.

Method. Retrospectively, 26 inpatients were identified over a two-month period who were prescribed antipsychotic drugs. Their notes were reviewed for documented weekly GASS scores for the duration of antipsychotic treatment. Initial data demonstrated $0 \%$ compliance with guidelines, as no patients completed a weekly GASS. The intervention to improve compliance was a training session for ward staff on implementing the GASS. Data were subsequently collected prospectively over three weeks for 15 patients.

Result. Seven patients completed the GASS weekly over three weeks, representing 47\% compliance. Two patients (13\%) completed two forms, three (20\%) completed one form, and three (20\%) completed no forms. There was a positive correlation between being offered the GASS and completing it - only one patient declined to complete it and was not offered it during the third week. Of the remaining 14 patients, if the GASS was offered there was $100 \%$ rate of completion. Staff did not offer the GASS to every patient each week, which accounted for most cases of non-completion. Some patients with pre-existing symptoms of physical illnesses included these on the GASS, which complicated interpretation. Future interventions could include further staff education, and involving a ward pharmacist to review results during medication reviews to optimise treatment compliance, as no medication changes resulted directly from patients completing the GASS. 
Conclusion. Compliance with completing the GASS weekly improved following staff education, identifying the main factor affecting compliance as staff not offering the GASS to patients. Patients generally engaged well with side-effect monitoring, as most completed the GASS when offered. Further staff education may produce even greater compliance with guidelines, and involving pharmacy staff to review GASS scores and inform medication choices may lead to use of the GASS resulting in more tolerable and effective treatment plans.

\section{Improving risk assessments for CAMHS admissions at Great Ormond Street Hospital}

Lisanne Stock ${ }^{\star}$ and Sacha Evans

Great Ormond Street Hospital

${ }^{\star}$ Corresponding author.

doi: $10.1192 /$ bjo.2021.316

Aims. During the COVID-19 pandemic, admissions to the Mildred Creek Unit (MCU), an Inpatient CAMHS Ward at Great Ormond Street Hospital (GOSH) changed.

The MCU is a 7-10 bed unit for children aged 7-15 years based on therapeutic milieu principles. The ward accepts patients via a planned national referral pathway, however, during the COVID-19 pandemic, patients were admitted as emergencies and consequently risk assessments were missed. Risk assessment is important in all admissions and as the MCU is not a locked unit, early risk assessment is particularly important.

We aimed to review whether risk assessment occurred within one working day of admission, as suggested by the ward risk assessment policy, and if this was not the case, our aim was to ensure that all risk assessments took place within this period via our audit interventions.

Method. We collated data looking at the time between admission to GOSH and the date at which first risk assessments took place. We then put in place three interventions.

1) Posters prompting doctors who were providing on-call liaison input to perform a risk assessment within one working day of admission.

2) New junior doctors were provided with written and verbal information to emphasise the importance of early risk assessment.

3) Guidelines also highlight that assessment of risk may need to be on-going. We therefore added a prompt section in the weekly ward round proforma with the aim of reducing the interval between risk assessments during admission.

The first audit cycle was conducted on the 3/8/2020 and the second on the $28 / 11 / 21$ to allow for a comparative number of inpatients between the first and second audit cycle.

Result. We found these interventions significantly reduced delays in risk assessments. Prior to the audit's first cycle the average delay between admission to GOSH/MCU and a risk assessment was 2 weeks. After the interventions there were no patients whose risk assessment was delayed outside the next working day parameters. Conclusion. This full cycle audit demonstrates the impact that prompts to clinical practice can make on patient care. It is important to recognise the need for flexible risk assessment with regular review, especially at times of clinical change. We hope that this continued trend for early risk assessment leads to improved clinical care and timely discussion of risk for all new CAMHS inpatients at GOSH.

\section{Audit of physical health monitoring in a forensic} psychiatric community caseload

Neeti Sud ${ }^{\star}$ and Michael Lacey

Cumbria, Northumberland, Tyne And Wear NHS Foundation Trust ${ }^{\star}$ Corresponding author.

doi: 10.1192/bjo.2021.317

Aims. Adherence to Cumbria Northumberland Tyne and Wear NHS Foundation (CNTW) Trust physical health monitoring guidelines for a caseload of community forensic psychiatry patients residing at Westbridge supported accommodation was audited to identify areas for improvement in practice. It was also our aim to highlight the delay in obtaining non-urgent investigations due to the need to minimize COVID infection transmission risks.

Method. Data were collected from mental health and acute trust electronic records (Rio and ICE) of all patients taking antipsychotic medications currently care coordinated by the Westbridge Forensic Community Mental Health Team (FCMHT) between January 2020 and January 2021 (8 patients). Analysis of compliance with standards set by Trust guidelines was made.

Result. In the chosen audit period, compliance with physical health monitoring standards was below target of $100 \%$ (80\% compliance for bloods, $50 \%$ for ECG). Reasons for non-compliance were unexpected restrictions in service availability (e.g. temporary closure of walk-in ECG clinic) and one omission of sending a prolactin levels request.

Conclusion. The need for practice adaptation and advance planning by team in anticipation of potential delays was identified. Request for routine bloods and ECGs will now be made two months before the annual due dates to compensate for delays in the new process with plan to continue re-audit yearly.

An audit cycle of physical health monitoring and record keeping of long term in-patients at male and female psychiatric rehabilitation wards using QI approach

Hina Tahseen* and Peter Bramall

Delfryn Lodge, Cygnet Health Care

${ }^{\star}$ Corresponding author.

doi: 10.1192/bjo.2021.318

Aims. To complete an audit cycle to evaluate and improve physical health monitoring practice for in-patients by incorporating small QI based projects between baseline audit and re-audit.

Background. People with mental health illness are at increased risk of physical illness, morbidity and mortality compared with general population, mainly due to adverse effects of psychotropic medications, polypharmacy, poor lifestyle choices and socioeconomic difficulties. It is important to recognise the need for active health promotion, including formal health checks for psychiatric in-patients.

Method. Standards were obtained from NICE Guidelines, RCPsych Report on Physical Health in Mental Health and Cygnet Health Care's Physical health policy.

An Audit tool with simple checklist was generated from key areas of Cygnet's physical health policy. Physical Health Files of 24 patients from Female Rehabilitation Ward and 28 patients from Male Rehabilitation Ward were audited in the initial audit cycle.

Checklist included physical health examination within 24 hours of admission, Annual Health Improvement Profile (HIP), 